\title{
Reversed Phase LC-UV Method Development and Validation for Simultaneous determination of three antiretrovirals: Lamivudine, Zidovudine, Nevirapine and Possible Degradants in a Fixed Dose Pharmaceutical Product
}

Anjali Joshi' ${ }^{1}$ and Moji Christianah Adeyeye ${ }^{2}$

\begin{abstract}
Background: Lamivudine (3TC), Zidovudine (AZT) and Nevirapine (NVP) are the common antiretrovirals prescribed in resource limited countries due to its cost effectiveness compared to other newer antiretrovirals. Monitoring quality of such generic versions of the dosage forms is a crucial aspect. The main aim of the study is to develop a simple reversed phase (RP) HPLC-UV method to determine 3TC, AZT, NVP with some of its degradation products cytosine and thymine that can be applied in monitoring the quality of such products.

Methods: A RP gradient HPLC method was developed using following chromatographic conditions: LunaC18 $150 \times 4.6 \mathrm{~mm}$ column; $50 \mathrm{mM}$ ammonium acetate buffer $(\mathrm{pH}=6.8)$ and methanol as mobile phase; Gradient mode of elution with $0-35 \% \mathrm{v} / \mathrm{v}$ methanol for first 10 minutes increased to $50 \% \mathrm{v} / \mathrm{v}$ till 12 minutes and isocratic to 18 minutes, return to initial at $20 \mathrm{~min}$; sample injection volume $50 \mu \mathrm{l}$; detection wavelength $265 \mathrm{~nm}$. Calibration $((n=6)$ and validation samples $(n=3)$ were prepared in triplicate for analysis and validated for specificity, linearity, range, accuracy and precision. The method was used in dissolution studies of the fixed dose combination tablets of AZT/3TC/NVP using USP Type I method.

Results: No interference between the analytes was observed. The method was found to be linear over the following concentration

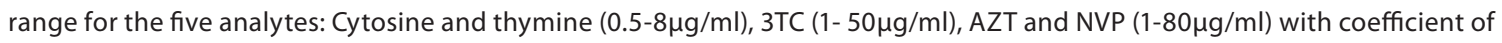
determination (R2) value $>0.9997$. Accuracy in terms of mean percent recovery for the five analytes was found to in the range of (Mean Recovery \pm S.D) $101.26 \pm 1.27 \%$. Intra-day and Inter-day precision (\%RSD) was found to be $0.49 \pm 0.28 \%$ and $2.24 \pm 0.26 \%$ respectively. There were no significant differences in the mean recovery of the APIs between the days as analyzed by two-way ANOVA at $p>0 \cdot 05$. No matrix effect due to the presence of other excipients in the formulation was observed in the chromatograms of the dissolution samples. Conclusion: The method developed was specific, accurate, reliable and can be applied during routine pharmaceutical analyses such as drug content analysis, dissolution studies and stability of the fixed dose combination product of AZT/3TC/NVP.
\end{abstract}

\section{Background}

Human Immunodeficiency Virus (HIV) has been one of the most devastating diseases affecting a large pediatric and adult population of the world. With the advent and development thereafter of various antiretrovirals, it has led to the significant reduction in the morbidity and mortality rates of the HIV infected population. The standard antiretroviral (ARV) therapy in treatment of these HIV-infections entails the use of combination drug therapy usually a triple combination such as two-nucleoside or nucleotide reverse transcriptase inhibitors (NRTIs) as a backbone and a non-nucleoside reverse transcriptase inhibitor (NNRTI) [1].

Out of various existing ARV drugs currently recommended by World Health Organization for HIV infection in pediatric population in resource limited countries, the combination of zidovudine/lamivudine (NRTI backbone) and nevirapine (NNRTI) is considered as one of the preferred ARV treatment $[2,3]$. Chemically lamivudine is (4-amino-1-[(2R,5S)-2(hydroxymethyl)-1,3-oxathio-lan-5-yl]-1,2dihydropyrimidine-2-one)

*correspondence: madeyeye@roosevelt.edu

${ }^{2}$ Department of Biopharmaceutical Sciences, College of

Pharmacy, Roosevelt University, Schaumburg, IL, USA.

Full list of Author information is available at the end of the article
(3TC) and Zidovudine is (1-[(2R,4S,5S)-4-azido-5-(hydroxymethyl) oxolan-2-yl]-5-methyl- 1,2,3,4-tetrahydropyrimidine-2,4-dione) (Figure 1). They are both nucleoside analogues possessing potent inhibitory activity against HIV reverse transcriptase. They are phosphorylated by kinases into the triphosphates, the pharmacologically active form. This active metabolite is incorporated into HIV DNA acting as a competitive inhibitor of reverse transcriptase leading to chain termination. Nevirapine is chemically 11-cyclopropoyl-5, 11-dihydro4-methyl-6H-dipyrido [3, 2-b: 2', 3'-e][1,4] diazepin-6-one (Figure 1). It is a non-competitive inhibitor DNA and RNA dependent polymerase that binds to a distant site from the reverse transcriptase enzyme's active site HIV-1 RNA to induce a conformational change at the active site and disrupt catalytic activity of the virus to transcribe viral RNA into DNA [4]. These combination drugs, when given as a fixed dose combination product rather than individual entities, has shown to improve therapy in terms of sustained virological suppression and significant reduction in the mortality rates of the HIV/AIDS infected people $[5,6]$.

Development of fixed-dose combinations is often associated with many design challenges such as drug-drug or drug-excipient interactions that could compromise the stability and efficacy of the 


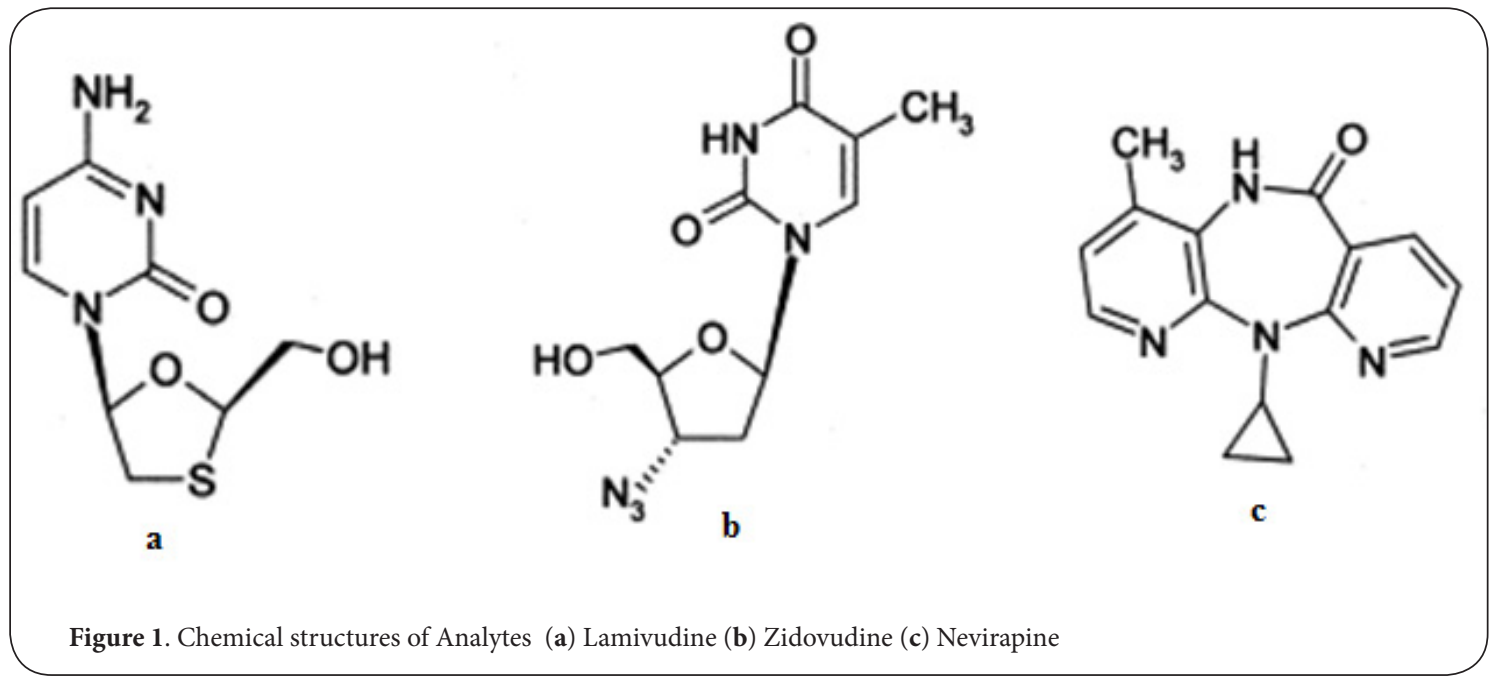

therapeutic agents. During the early phase of pharmaceutical product development, it's essential to investigate the stability of the drug substance and its compatibility with other drugs (in case of multiactives formulations) and different excipients which could affect the overall quality of the product in terms of its safety and performance. [7]. ICH guidelines on stability of the new drug substance and drug products Q1A (R2) also emphasize the aforementioned statement stating "information on the stability of the drug substance as an integral part of the systematic approach to stability evaluation"[8]. Hence, during the process of the development of such fixed dose combination products, it is imperative to have a simple stability indicating analytical method that can be used easily to (a) monitor the possible drug's chemical instability in preformulation studies such as drug/drug or excipient compatibility studies and (b) perform the various in-process/end process product evaluation such as monitoring potency and dissolution of products during the early product development phases.

Various HPLC analysis methods have been reported for the analysis of different NRTIs and NNRTIs but there is lack of a simple, cost effective, definitive stability indicating method for analyzing the combination of 3TC, AZT and NVP that can be used in a FDC formulation [9-13]. Hence, the main objective of this study is to develop a simple and robust stability indicating HPLC-UV method for the simultaneous determination of the three analytes together with their possible degradants.

According to literature, forced degradation studies of zidovudine and lamivudine under different hydrolytic conditions resulted in thymine and cytosine as the major degradation products shown in Figures $2 A$ and $2 B$ respectively $[14,15]$. These results were concurrent with the results of similar force degradation studies conducted in our laboratory for zidovudine and lamivudine to observe their possible degradants. During the LC method development, forced degradation studies were conducted for zidovudine, lamivudine and nevirapine in various $\mathrm{pH}$ and temperature conditions. Individual drugs were subjected to stress at acidic, alkaline and neutral $\mathrm{pH}$ at different conditions to monitor the hydrolytic degradation. 3TC was found to degrade into cytosine in alkaline $\mathrm{pH}$ and zidovudine into thymine at acidic $\mathrm{pH}$ when stressed at high temperature. Cytosine and thymine was confirmed to be the degradation product by comparing the chromatograms between the stressed sample and the sample containing the pure degradant itself. Nevirapine was found to be relatively stable without any degradation peaks. These results were consistent with the data reported in the literature. Based on the forced degradation and the major degradants or the possible impurities detected, a stability indicating RP-HPLC-UV method was developed and validated. This method hence would be applicable in the regular pharmaceutical analysis of the products containing either one of these or all of these active components.

\section{Methods}

Chemicals and Reagents:

Zidovudine, lamivudine and nevirapine were purchased from Dayang Chemicals, China. Cytosine and Thymine were purchased from Sigma Aldrich Company, USA. HPLC grade Methanol was purchased from Fischer Scientific, USA. Ammonium acetate was sourced from Fischer scientific, USA). Water used during the analysis was purified using Milli-Q purification unit in-house. All other chemicals used were of pharmaceutical and analytical grade.

\section{Instrument:}

High performance liquid chromatography (HPLC) method was developed and performed using Waters HPLC 2690 separation module equipped with auto sampler and Waters 996 PDA detector (scanning in the UV range of $200-400 \mathrm{~nm}$ ). All the chromatograms were analyzed at a single wavelength of $265 \mathrm{~nm}$ and the data were handled using EMPOWER ${ }^{\text {TM }} 2.0$ software.

\section{Chromatographic Conditions:}

The chromatographic conditions for the analysis of five analytes viz thymine, cytosine, lamivudine, zidovudine and nevirapine are reported as follows:

Colum: Luna C18 column (150*4.5mm) 5microns; Mobile phase: 

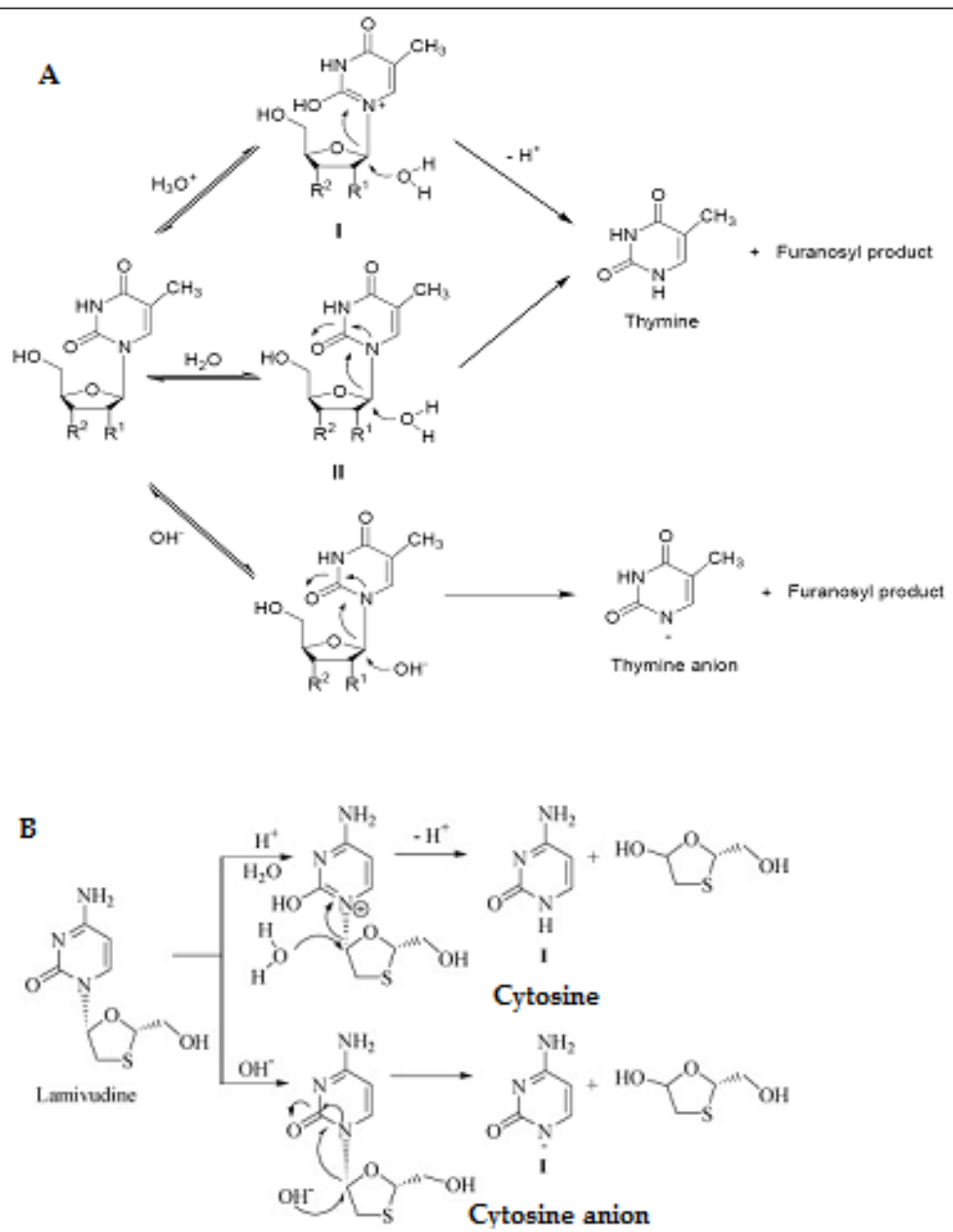

Figure 2. Degradation pathways for AZT and 3TC in acidic and alkaline conditions A: Zidovudine [Adapted from ref 12], B: Lamivudine [Adapted from ref 13].

Organic Phase: A: Methanol ;Aqueous Phase: B: 50mm Ammonium phosphate ( $\mathrm{pH}=6.82)$; Gradient Mode of Elution: First 10 minutes: organic phase $0-35 \% \mathrm{v} / \mathrm{v}, 12 \mathrm{~min}-18 \mathrm{~min} 50 \% \mathrm{v} / \mathrm{v}$. Flow rate: $1 \mathrm{ml} / \mathrm{min}$; Sample Injection volume: $50 \mu \mathrm{l}$.

Preparation of calibration standard and quality control (QC) samples: For calibration standards, the stock solution for zidovudine, lamivudine, their respective degradation products cytosine and thymine was prepared in purified water $(18.2 \mathrm{M} \Omega \mathrm{cm}$ of electrical resistivity). Nevirapine was prepared in water: methanol: 50:50 as solvent. The stock solutions were then suitably diluted in the working concentration range using $50 \mathrm{mM}$ aqueous buffer of ammonium acetate $(\mathrm{pH}=6.82)$. Samples were prepared for the five analytes in the concentration range as follows: cytosine and thymine $(0.5-8 \mu \mathrm{g} /$ $\mathrm{ml})$, lamivudine (1-50 $\mathbf{\mu g} / \mathrm{ml})$, zidovudine and nevirapine (1-80 $\boldsymbol{\mu g}$ / $\mathrm{ml}$ ) respectively to develop a calibration curve. The concentration ranges of the analytes were chosen based on the dissolution studies of fixed dose combination formulation of zidovudine/lamivudine/ nevirapine 60/30/55 mg tablets. All the samples were prepared in triplicate for the six calibration points. Similarly, a separate set of stock solutions were prepared for the QC samples intended for its use in method validation for determining accuracy and precision. All the QC samples at three different concentration ranges were prepared in triplicates. Fresh calibration and QC samples were prepared in different days.

\section{Method Validation:}

The method thus developed was validated for all the five analytes in compliance with ICH guidelines for the following parameters: (a) Selectivity and Specificity (b) Linearity and Range (c) Accuracy and Precision (d) Ruggedness (\% Recovery \pm SD) and (e) Limit of Detection (LOD) and Limit of Quantitation (LOQ) [16].

(a) Selectivity and Specificity: Selectivity is the ability to measure accurately and specifically the analyte in the presence of components that may be expected to be present in the sample matrix. Specificity refers to the ability to assess the unequivocally the analyte in presence of other components. It ensures that the signal measured comes from the substance of the interest and that there is no interference 
from excipient and/or degradation products and/or impurities. This was determined by assessing the peak identity and purity. Peak purity angle that is less than peak threshold angle is an indication of spectral homogeneity or purity of the drug.

(b). Linearity and Range: A six point calibration curve was constructed by analyzing the samples in the concentration range as mentioned above. Linearity was measured for the selected concentration range by fitting the linear regression model and assessing the coefficient of determination $\left(\mathrm{R}^{2}\right)$. The residual plots for calibration curve of each analytes were also evaluated to see if there are any patterns.

(c). Accuracy and Precision: Accuracy and precision of the method were evaluated analyzing the validation samples $(n=9)$ at three different levels in triplicates of concentration (high, medium and low). To measure the repeatability or the method precision, samples were analyzed at three different concentration levels (low, medium and high) in triplicates. The recovery was then calculated and precision is reported as relative standard deviation (RSD) for these values. Intermediate precision was evaluated by repeating the experiment using freshly prepared samples on three separate days. The data was analyzed by means of two-way analysis of variance (ANOVA) at level of significance set at $a=0.05$. P-value $>0.05$ would indicate no significant differences in the results between days or different levels of concentration.

(d). Ruggedness: Method ruggedness was evaluated based on the recovery of the analytes at three different concentrations: low, medium and high levels.

(e). $L O D$ and LOQ: The detection limits (DL) and the quantitation limits for all the analyte were calculated using the following equations:

$L O D=3.3 \mathrm{\sigma} / \mathrm{S}$ and $L O Q=10 \mathrm{\sigma} / \mathrm{S}$

where $\sigma=$ the standard deviation of the response as estimated from the standard deviation of $y$-intercepts of regression lines and $S=$ the mean slope of the calibration curves of the analyte.

Application of the analytical method: The method developed was applied in (a) preformulation studies- drug-drug or drug excipient interaction (b) determining the drug content analysis and (c) dissolution studies of the product developed with these actives. Three different batches of fixed dose combination tablets containing 60/30/50mg of AZT/3TC/NVP were compressed in house (Manufacturing laboratory, Duquesne University using Elizabeth HATA tablet press). Tablets were stored in HDPE plastic bottles after compaction.

Drug-Drug and Drug excipient interaction studies: The method was applied to monitor any chemical degradation during the preformulation studies conducted prior to the fixed dose combination formulation development. The chemical degradation was followed by isothermal stress testing protocol [17]. Individual drugs and drug mixtures (in the ratio of 3TC: AZT: NVP: 1:2:1.8) were kept in the closely tight scintillating vial. The samples were prepared and kept in dry condition and mixed with $5 \%$ water. The mixtures in the vials were then stressed at $50^{\circ} \mathrm{C}$ for 3 weeks using an isotemp oven. Similar samples containing individual drugs and drug mixtures were also kept in room temperature as a control or reference material. The drugs from the samples were then extracted in $80 \% \mathrm{v} / \mathrm{v}$ methanol in water by sonicating for 30 minutes. Suitable dilutions were made and the final dilution was filtered prior to HPLC analysis to assess the drug recoveries. Samples both in stressed condition and controls were prepared in triplicates. Also, samples containing the select excipients as chosen from the excipient screening test were mixed with the individual drug and drug mixtures and stressed, and the drugs were similarly extracted.

Drug content Analysis: For drug content analysis of tablets containing 30:60:50mg: 3TC/AZT/NVP, six tablets were randomly selected from different batches. Individual tablet was weighed and dispersed in $25 \mathrm{ml}$ of methanol water solution $(50 \% \mathrm{v} / \mathrm{v})$ and sonicated for 30 minutes. Suitable dilutions were performed sequentially with ammonium buffer to achieve the analyte concentration within the range of calibration points. The final dilution was filtered using $0.45 \mu$ m nylon filter prior to HPLC analysis. Drug content of the tablets $(n=6)$ was determined based on the recovery of the analytes from the samples and is expressed as the percentage amount of assay value.

To determine assay value, twenty random tablets were taken, weighed and grinded into powder using mortar and pestle. An aliquot of powder equivalent to one tablet was weighed and dispersed in $25 \mathrm{ml}$ of methanol water solution $(50 \% \mathrm{v} / \mathrm{v})$ and sonicated for 30 minutes. Suitable dilutions were made to achieve a concentration range within the calibration points. The samples were prepared in triplicates.

Dissolution Studies: Dissolution or drug release from the tablets $(n=6)$ was determined using USP Type II method using Vankel dissolution tester. Six tablets randomly selected from each batches, were placed in baskets and immersed in dissolution vessel $(n=6)$ containing phosphate buffer $(\mathrm{pH}=2.0)$. The temperature of the medium was maintained at $37.0 \pm 0.50 \mathrm{C}$ and the basket speed was maintained at 100RPM. Samples were withdrawn at 5, 10, 20, 30, 45 and 60 minutes. The samples were filtered and subjected to HPLC analysis.

\section{Results \& Discussion}

Selectivity and Specificity: Representative chromatogram of the analysis is shown in Figure 3. No interference between the five analytes zidovudine, lamivudine, nevirapine, cytosine and thymine was observed based on the retention time as represented in Table 1, indicating the selectivity and specificity of the method. In addition, the retention times for all the analytes remain the same throughout the different concentrations used for the calibration. Furthermore, the peak purity angles were consistently less than threshold angle for all five analytes at both low and high concentrations confirming the specificity of the method.

Linearity and Range: The range for the concentration of the five different analytes and the corresponding $\mathrm{R}^{2}$ value for each of them are reported in Table 1. The regression coefficient values those are greater than 0.999 indicated a good linearity in the concentration range chosen. The residual plots of the observed vs. predicted peak areas for all the analytes did not indicate any significant pattern supporting the linearity of the method. 


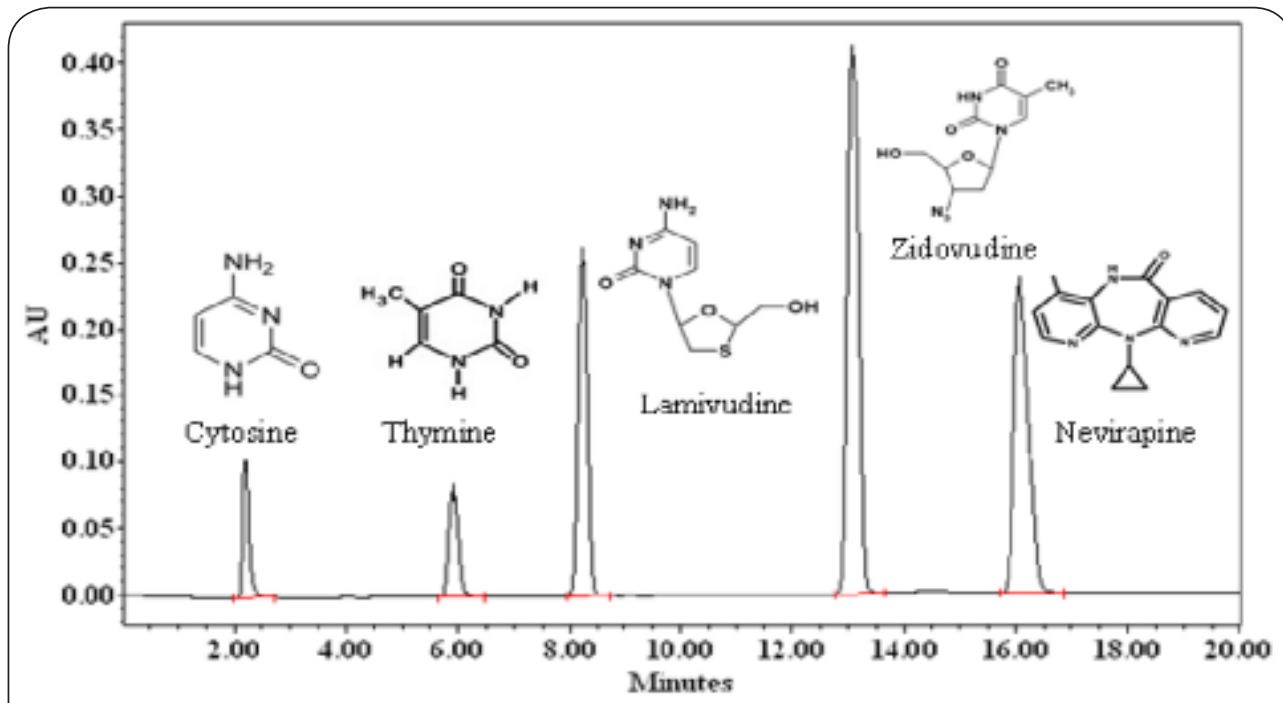

Figure 3. A representative chromatogram showing the separation of the five analytes

\begin{tabular}{|c|c|c|c|c|c|c|c|c|}
\hline \multirow[b]{2}{*}{ Analyte } & \multirow{2}{*}{$\begin{array}{l}\text { Retention } \\
\text { Time (min) }\end{array}$} & \multicolumn{2}{|c|}{ Linearity and Range } & \multirow{2}{*}{$\begin{array}{l}\text { Accuracy } \\
\text { Recovery } \\
(\% \pm \text { S.D })^{*}\end{array}$} & \multicolumn{2}{|c|}{ Precision } & \multicolumn{2}{|c|}{$\begin{array}{c}\text { Limits of Detection /Quanti- } \\
\text { tation }\end{array}$} \\
\hline & & $\begin{array}{c}\text { Response } \\
\text { range }(\mu \mathrm{g} / \\
\mathrm{ml})\end{array}$ & $\mathbf{R} 2$ & & $\begin{array}{l}\text { Intra day Pre- } \\
\text { cision } \S(\%)\end{array}$ & $\begin{array}{l}\text { Interday Pre- } \\
\text { cision\$\$ (\%) }\end{array}$ & $\begin{array}{l}\text { LOD } \\
\left(\mu \mathrm{gml}^{-1}\right)\end{array}$ & $\begin{array}{c}\text { LOQ } \\
\left(\mu \mathrm{gml}^{-1}\right)\end{array}$ \\
\hline $\mathrm{C}$ & $2.20 \pm 0.02$ & $0.5-8.0$ & $\begin{array}{c}0.9999 \pm \\
0.0012\end{array}$ & $100.71 \pm 1.85$ & 0.77 & 2.02 & 0.18 & 0.50 \\
\hline $\mathrm{T}$ & $5.70 \pm 0.01$ & $0.5-8.0$ & $\begin{array}{c}0.9997 \pm \\
0.0015\end{array}$ & $102.23 \pm 4.86$ & 0.63 & 2.00 & 0.02 & 0.07 \\
\hline $\mathrm{L}$ & $7.90 \pm 0.01$ & $1.0-50.0$ & $\begin{array}{c}0.9997 \pm \\
0.0020\end{array}$ & $100.15 \pm 2.09$ & 0.03 & 2.12 & 0.26 & 0.79 \\
\hline $\mathrm{Z}$ & $12.80 \pm 0.01$ & $1.0-80.0$ & $\begin{array}{c}0.9998 \pm \\
0.0006\end{array}$ & $100.25 \pm 3.06$ & 0.58 & 2.55 & 0.07 & 0.22 \\
\hline $\mathrm{N}$ & $15.70 \pm 0.00$ & $1.0-80.0$ & $\begin{array}{c}0.9998 \pm \\
0.0006\end{array}$ & $103.00 \pm 5.05$ & 0.46 & 2.49 & 0.21 & 0.65 \\
\hline
\end{tabular}

Table 1. Summary of the validation parameters

${ }^{*} \mathrm{n}=9$; : No significant differences between the different concentration levels, $\mathrm{p}>0.05$; $\$$ s: no significant differences between the days, $\mathrm{p}>0.05$

Accuracy and Precision: Accuracy expresses the closeness of agreement between the value found and the value that is accepted as either a conventional true value or an accepted reference value. It is expressed in terms of the recovery of the analytes. Recoveries were evaluated for three different working concentrations in the low, medium and high levels. The recovery values with the associated standard deviation are reported in the Table 1.

Method Precision (Repeatability) evaluates the variation experienced by a single analyst on a single instrument. Intermediate precision refers to variations within a laboratory such as different days, different instruments and different analyst. Variations between days at three different levels were evaluated in the method development. All the method precision and the intermediate precision values are reported in the Table 1. Precision values less than $5 \%$ indicated the method was precise in evaluating the analytes at the chosen concentration range.

Ruggedness: Method ruggedness as evaluated based on the recovery of the analytes at three different concentrations: low, medium and high levels indicated that the recovery values for all the five analytes were greater than $95 \%$ indicating the method is rugged (Table 2).

LOD and LOQ: The detection and quantitation limits were evaluated for all the five analytes are summarized in Table 1. The lowest limit of quantitation for lamivudine, zidovudine and nevirapine were found to be $0.79 \mu \mathrm{gml}^{-1}, 0.22 \mathrm{\mu gml}^{-1}$ and $0.65 \mathrm{mgml}^{-1}$ respectively, indicating a good sensitivity of the method.

Application of the analytical method: No matrix effect, i.e. no interference in the response or signal of zidovudine, lamivudine and nevirapine, due to the presence of other excipients in the formulation was observed in the chromatograms of the analyzed stressed, dissolution and drug content analysis samples. A sample chromatogram obtained from the dissolution studies is presented (Figure 4). This is an indication of the suitability of this developed method in the regular pharmaceutical analysis of the formulation containing these actives. 


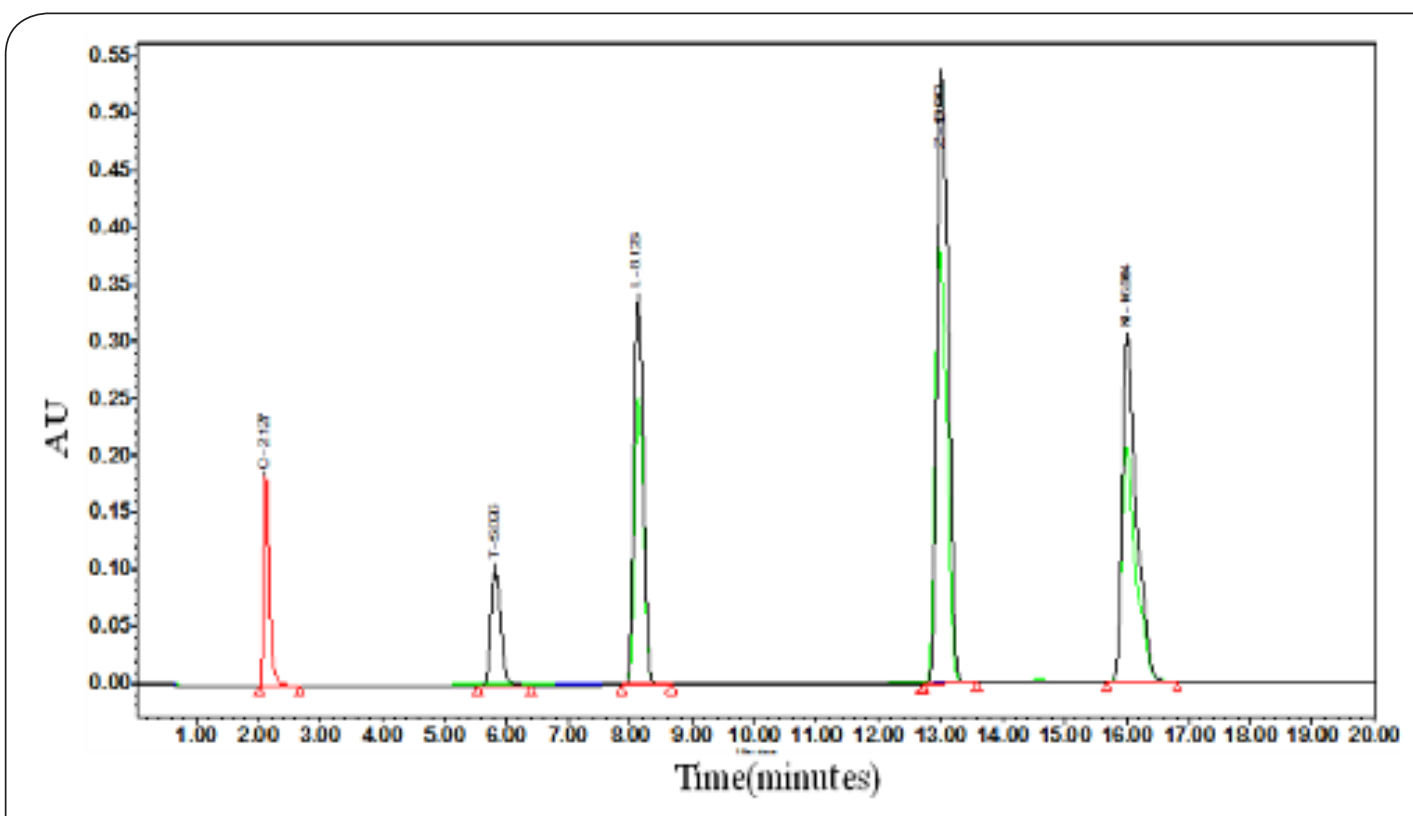

Figure 4. A representative chromatogram (overlayed) showing no matrix effect from a dissolution sample: Black line: Sample of pure analytes; Green line: Analytes from the matrix solution obtained by dissolving tablets

\begin{tabular}{ccccccc}
\hline \multirow{2}{*}{$\begin{array}{c}\text { Concentra- } \\
\text { tion Levels }\end{array}$} & \multicolumn{5}{c}{ \% Recovery \pm SD } & \multirow{2}{*}{ Day } \\
\cline { 2 - 6 } & Cytosine & Thymine & Lamivudine & Zidovudine & Nevirapine & \\
\hline Low & $97.29 \pm 1.27$ & $104.79 \pm 1.46$ & $99.97 \pm 0.07$ & $103.21 \pm 1.71$ & $106.69 \pm 1.24$ & \\
Med & $98.97 \pm 0.37$ & $99.35 \pm 0.28$ & $99.87 \pm 0.01$ & $100.84 \pm 0.06$ & $96.51 \pm 0.16$ & 1 \\
High & $99.42 \pm 0.63$ & $101.58 \pm 0.24$ & $100.52 \pm 0.00$ & $99.86 \pm 0.01$ & $98.95 \pm 0.05$ & \\
\hline Low & $101.79 \pm 1.00$ & $107.28 \pm 2.14$ & $104.14 \pm 0.44$ & $97.69 \pm 0.77$ & $110.65 \pm 1.73$ & \\
Med & $102.30 \pm 0.13$ & $98.91 \pm 0.30$ & $99.01 \pm 0.02$ & $102.39 \pm 0.02$ & $99.68 \pm 0.03$ & 2 \\
High & $103.17 \pm 0.65$ & $97.21 \pm 0.19$ & $98.26 \pm 0.03$ & $101.72 \pm 0.03$ & $98.86 \pm 0.04$ & \\
\hline Low & $101.67 \pm 0.73$ & $112.00 \pm 0.51$ & $97.47 \pm 0.99$ & $94.17 \pm 2.74$ & $109.74 \pm 0.77$ & \\
Med & $100.57 \pm 0.05$ & $98.63 \pm 0.47$ & $102.63 \pm 0.13$ & $103.80 \pm 0.05$ & $102.96 \pm 0.06$ & 3 \\
High & $101.16 \pm 0.20$ & $100.29 \pm 0.18$ & $99.47 \pm 1.83$ & $98.59 \pm 0.05$ & $102.94 \pm 0.02$ & \\
\hline
\end{tabular}

Table 2. Ruggedness (\% Recovery \pm SD)

${ }^{\star}$ All the experiment at different concentration levels were performed in triplicates.

Preformulation Studies: Recoveries of the drugs from stressed samples were greater than $97 \%$. For instance, the recoveries ( \pm SD) of drugs 3TC, AZT and NVP from the stressed samples containing all the three drugs and formulation components, with $5 \% \mathrm{w} / \mathrm{w}$ of water stored at $50^{\circ} \mathrm{C}$ for three weeks were found to be $99.33(0.01), 101.04(0.36)$ and $97.38(2.74)$ respectively. This indicates the suitability of the method in quantitation of the analytes in stressed samples. However, the samples did not show any indication of degradation products as compared to the solution state forced degradation studies samples. This may be attributed to the degree of the stress applied to the samples and the kinetics of the reaction. The study did not involve the kinetic study of the degradation rates of the drug components, hence this could be a research area that can be explored in detail to understand and model the degradation rates of 3 TC, AZT and NVP particularly.
Drug Content Analysis: Analysis of drug content in tablets from three different batches resulted in the estimation of the drugs in the tablets which was close to the theoretical amount or label claim (Table 3). These values comply with the USP pharmacopeia assay specifications for active components i.e. $90.0-110.0 \%$, which is required to be met by most drug formulations. This validates the usefulness of the proposed techniques in simultaneously determining the three antiretrovirals as a part of routine quality control analysis of generic drug combinations of these drugs.

\begin{tabular}{cccc}
\hline \multirow{2}{*}{ Batches } & \multicolumn{3}{c}{ Drug content [\% recovery \pm SD (RSD)]* } \\
\cline { 2 - 4 } & ZDV & 3TC & NVP \\
\hline B1 & $98.32 \pm 2.25(2.52)$ & $99.08 \pm 2.66(2.95)$ & $101.10 \pm 1.64(1.78)$ \\
B2 & $99.17 \pm 1.74(1.93)$ & $99.77 \pm 2.69(2.96)$ & $101.30 \pm 2.78(3.02)$ \\
B3 & $99.11 \pm 1.47(1.63)$ & $101.44 \pm 1.83(1.98)$ & $102.77 \pm 0.97(1.04)$ \\
\hline \multicolumn{2}{l}{ Table 3. Drug content Analysis }
\end{tabular}

${ }^{*}$ All results were an average of six samples. 


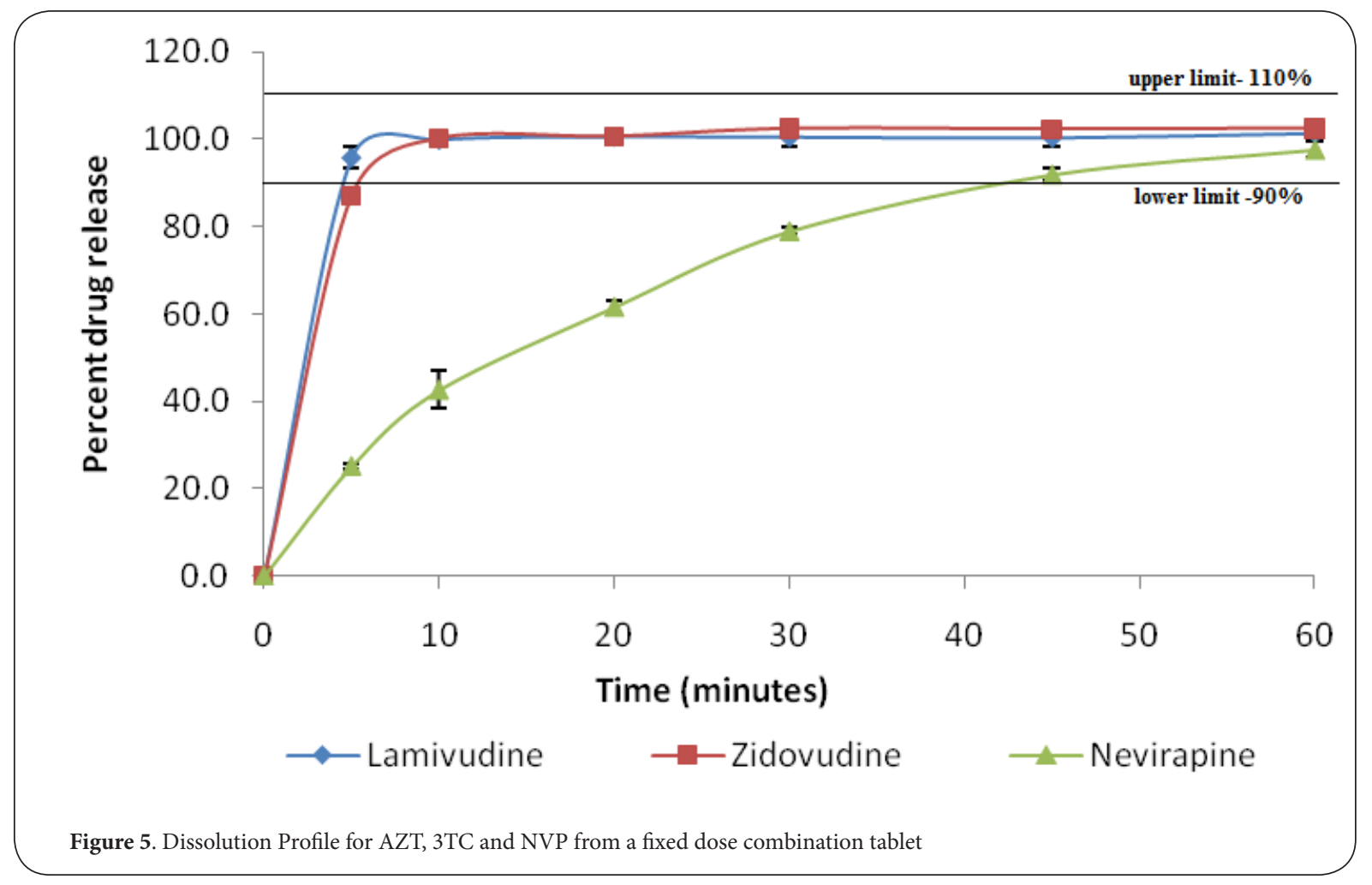

Dissolution Studies: Similarly, dissolution studies indicated that more than $80 \%$ of 3 TC and AZT was released within 5 minutes. Similarly, more than $70 \%$ of NVP was released within 30 minutes. All the drug release profile met the USP specifications as indicated in individual drug monographs (Figure 5).

The developed method offers a few advantages over the method that have been reported in literature. First is the cost effectiveness of the system as methanol is the only organic solvent that has been used for the method development. Other systems mostly used acetonitrile as the major organic solvent and in some cased even used a combination of various organic solvents and phosphate buffer which would increase the operating cost if such analysis has to be performed in a regular basis $[9,13,18-20]$. Methanol is cheaper, supposedly less damaging to health. Additionally, methanol is a polar organic solvent and hence reduces the risk of salt precipitating in narrow capillaries which in turn improve the operation efficiency of the HPLC unit with moderate back pressure for prolong periods. Second is the simplicity of the method. Ion pairing reagent such as octane sulphonic acid was used in some investigation [21]. Use of such reagents would affect the column life as it is never totally washed from the column and also when used in the method, it generally takes lots of more column volumes of mobile phase to equilibrate the column. Hence, the method developed surpasses these problems and hence can be used easily for the routine analysis.

\section{Conclusions}

A relatively new and simple HPLC-UV method was developed for the simultaneous determination of the three antiretrovirals: zidovudine, lamivudine and nevirapine - together with their associated degradants. The method developed and validated according to the $\mathrm{ICH}$ guidelines was specific, accurate, and reliable and can be applied in regular pharmaceutical analysis of formulations containing 3TC, AZT and NVP and their degradation products.

\section{Competing interest}

The author(s) declare that they have no competing interests.

\section{Author's contributions}

AJ carried out all the experiments, performed data analysis and drafted the manuscript. MCA is research advisor and supervisor to $\mathrm{AJ}$; she conceived the project idea and led the development of fixed dose combination tablets. All authors have read and approved the final manuscript.

\section{Author's information}

${ }^{1}$ Graduate School of Pharmaceutical Sciences, Mylan School of Pharmacy, Duquesne University, Pittsburgh, PA, USA.

\section{Article history}

Editor's: Hassan Y. Aboul Enein, National Research Centre, Egypt. Leena Suntornsuk, Mahidol University, Thailand.

Received: 28-Feb-2012 Revised: 01-Apr-2012

Accepted: 14-Apr-2012 Published: 23-Apr-2012 


\section{References}

1. 2009. Guidelines for the use of Antiretroviral agents in Pediatric HIV infection, developed by working group on antiretroviral therapy and medical management of HIV infected children convened by the National resource center at the Francois Xavier bagnoud center, UMDJ, the health resources and services administration (HSRA) and the National Institutes of health $(\mathrm{NIH})$.

2. WHO. 2006. World Health Organization. Pediatric guideline on Antiretroviral theray of HIV infection in infants and children in resource limited settings: towards universal access.

3. WHO. 2009. Towards universal access: scaling up priority HIV/AIDS interventions in the health sector: progress report

4. Brunton LL, Chabner BA, Knollmann BC, eds. 2011. Goodman \& Gilman's The Pharmacological Basis of Therapeutics

5. Katlama C, Ingrand D, Loveday C, Clumeck N, Mallolas J, Staszewski S, et al.: Safety and efficacy of lamivudine-zidovudine combination therapy in antiretroviral-naive patients. A randomized controlled comparison with zidovudine monotherapy. Lamivudine European HIV Working Group. JAMA 1996; 276;(2.);118-25. | Pubmed

6. Palella FJ, Jr., Delaney KM, Moorman AC, Loveless MO, Fuhrer J, Satten $\mathrm{GA}$, et al.: Declining morbidity and mortality among patients with advanced human immunodeficiency virus infection. HIV Outpatient Study Investigators. N Engl J Med 1998; 338;(13.);853-60. | Article | | Pubmed

7. 2005. ICH guideline:Q8-Pharmaceutical Development.

8. 2003. ICH Guideline: Q1A (R2)-Stability Testing of New Drug Substances and Products.

9. Anantha Kumar D, Naveen Babu MN, Seshagiri Rao JVLN, Jayathirth Rao V. 2010: Simultaneous determination of lamivudine, zidovudine and nevirapine in tablet dosage forms by RP-HPLC method. Rasayan Journal of Chemistry 3:94-9.

10. Dunge A, Sharda N, Singh B, Singh S: Validated specific HPLC method for determination of zidovudine during stability studies. J Pharm Biomed Anal 2005; 37;(5.);1109-14. | Article | Pubmed

11. Kapoor N, Khandavilli S, Panchagnula R: Simultaneous determination of lamivudine and stavudine in antiretroviral fixed dose combinations by first derivative spectrophotometry and high performance liquid chromatography. J Pharm Biomed Anal 2006; 41;(3.);761-5. | Article | Pubmed

12. Kapoor N, Khandavilli S, Panchagnula R. 2006. Simultaneous determination of lamivudine, stavudine and nevirapine in antiretroviral fixed dose combinations by high performance liquid chromatography. Analytica Chimica Acta 570:41-5.

13. Solomon G, Hymete A, Mohamed AMI, Bekhit AA. 2010. HPTLCdensitometric method development and validation for simultaneous determination of lamivudine, nevirapine and zidovudine in fixed dose combinations. Thai Journal of Pharmaceutical Sciences 35:77-88. | Article I

14. Bedse G, Kumar V, Singh S: Study of forced decomposition behavior of lamivudine using LC, LC-MS/TOF and MS(n). J Pharm Biomed Anal 2009; 49;(1.);55-63. | Article | Pubmed

15. Dunge A, Chakraborti AK, Singh S: Mechanistic explanation to the variable degradation behaviour of stavudine and zidovudine under hydrolytic, oxidative and photolytic conditions. J Pharm Biomed Anal 2004; 35;(4.);965-70. | Article | Pubmed

16. ICH. 1996. Validation of analytical procedures: Methodology.

17. Serajuddin AT, Thakur AB, Ghoshal RN, Fakes MG, Ranadive SA, Morris $\mathrm{KR}$, et al.: Selection of solid dosage form composition through drugexcipient compatibility testing. J Pharm Sci 1999; 88;(7.);696-704. | Article I Pubmed

18. Hari Krishnan N, Gunasekaran V, Roosewelt C, Meher VK, Kalaivani K, Ravichandiran V. 2008. Simultaneous estimation of lamivudine, zidovudine and nevirapine by RP-HPLC in pure and pharmaceutical dosage form. Asian Journal of Chemistry 20:2551-6.

19. Kayitare E, Vervaet C, Ntawukulilyayo JD, Seminega B, Bortel V, Remon JP: Development of fixed dose combination tablets containing zidovudine and lamivudine for paediatric applications. Int J Pharm 2009; 370;(1-2.);41-6. | Article | Pubmed

20. Lavra ZMM, Neto PJR, Da Silva RMF, De Medeiros FPM. 2008. Development and validation of an analytical method for simultaneous determination of fixeddose combination tablets of lamivudine, zidovudine and nevirapine by high performance liquid chromatography. Quim. Nova 31:969-74.

21. Fan B, Stewart JT: Determination of zidovudine/lamivudine/nevirapine in human plasma using ion-pair HPLC. J Pharm Biomed Anal 2002; 28;(5.);903-8. | Article | Pubmed

Citation:

Joshi A and Adeyeye M C: Reversed Phase LC-UV Method Development and Validation for Simultaneous determination of three antiretrovirals: Lamivudine, Zidovudine, Nevirapine and Possible Degradants in a Fixed Dose Pharmaceutical Product. journal of Pharmaceutical Technology and Drug Research 2012, 1:4. http://dx.doi.org/10.7243/2050-120X-1-4 